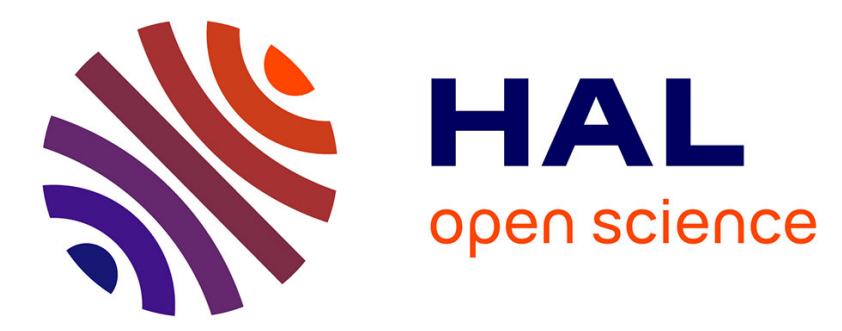

\title{
Minimization of the rate of change in torques during contact transitions for humanoids
}

Yang Tan, Darwin Lau, Mingxing Liu, Philippe Bidaud, Vincent Padois

\section{To cite this version:}

Yang Tan, Darwin Lau, Mingxing Liu, Philippe Bidaud, Vincent Padois. Minimization of the rate of change in torques during contact transitions for humanoids. European Control Conference, Jun 2016, Aalborg, Denmark. hal-01398737

\section{HAL Id: hal-01398737 \\ https://hal.science/hal-01398737}

Submitted on 17 Nov 2016

HAL is a multi-disciplinary open access archive for the deposit and dissemination of scientific research documents, whether they are published or not. The documents may come from teaching and research institutions in France or abroad, or from public or private research centers.
L'archive ouverte pluridisciplinaire HAL, est destinée au dépôt et à la diffusion de documents scientifiques de niveau recherche, publiés ou non, émanant des établissements d'enseignement et de recherche français ou étrangers, des laboratoires publics ou privés. 


\title{
Minimization of the rate of change in torques during contact transitions for humanoids
}

\author{
Yang $\operatorname{Tan}^{1}$, Darwin Lau ${ }^{1}$, Mingxing Liu ${ }^{1}$, Philippe Bidaud ${ }^{1,2}$ and Vincent Padois ${ }^{1}$
}

\begin{abstract}
Humanoid robots are commonly required to make contacts with the environment in order to perform various tasks. For tasks such as walking, sudden establishment and breaking of contacts are unavoidable. Such behaviours may result in large changes in actuator torques ( lashing), leading to potential control instability and damage to the actuators. In this paper, an approach to minimise sudden changes in actuation torques is proposed. This is achieved using Model Predictive Control (MPC) to modify the maximum and minimum allowable forces for a reactive whole-body controller. The MPC previews the evolution of contacts in time and generates smoother maximum and minimum allowable forces. The effect of the proposed approach is simulated for different scenarios on the iCub robot, such as standing up from a sitting posture and the lifting and lowering of a foot while standing. The results show that the proposed approach significantly decreases the changes in joint torques at the instances when contacts are broken or established.
\end{abstract}

\section{INTRODUCTION}

Free-floating robots, such as humanoids, are required to make contacts with the environment. For example, when the robot stands on the ground, contacts are intrinsically required for balance (see Figure 1). Contacts are usually treated as constraints in the control problem [1]-[3] and are expressed in two parts: one is the friction cone constraint [4,5]; and the other is the linear complementary condition [6]. During the walking motion, contacts between the feet and the ground must be established and broken in order to move around in the environment. At the instance when a contact is broken, the contact force decreases to zero. On the contrary, when a contact is established, a contact force may suddenly increase from zero. The sudden addition and removal of contact constraints can result in a discontinuous control signal with potentially dangerous effects: 1) damage to the actuators; 2) bad control performances; and 3) damage to the environment. Despite these consequences, the problem of sudden changes of contacts has not been well considered in the literature.

To prevent large sudden changes in actuation torques, one possible approach is to prevent the large changes of contact forces in the controller. The approach proposed in [7] aims at gradually decreasing the normal contact force to zero before the contact is broken. This is achieved by explicitly regulating the contact force as a desired task of the controller. While this approach provides interesting results, it is strongly related to the method chosen to describe and solve task hierarchies. In that respect, it does not provide a generic way of dealing with torque discontinuities

\footnotetext{
${ }^{1}$ Yang Tan, Darwin Lau, Mingxing Liu, Philippe Bidaud and Vincent Padois are with:

Sorbonne Universités, UPMC Univ Paris 06, CNRS, Institut des Systèmes Intelligents et de Robotique (ISIR), 4 place Jussieu, 75005 Paris, France

Email: $\{\tan , 1 \mathrm{au}, \mathrm{liu}$, bidaud, padois\}@isir.upmc. fr

${ }^{2}$ Philippe Bidaud is with the ONERA, 91123 Palaiseau, France

Email:philippe.bidaudeonera.fr
}

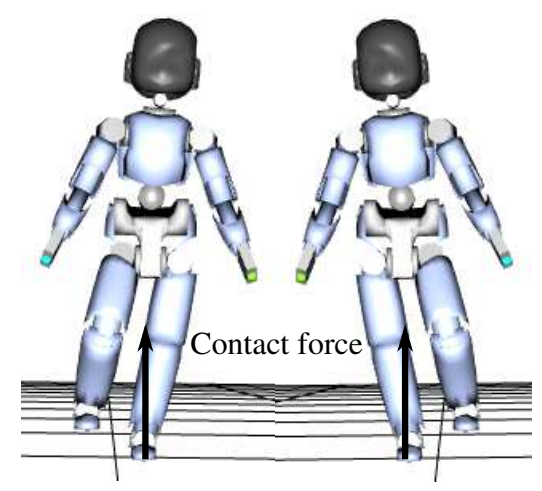

Fig. 1: Humanoid robot maintaining balance through single support contact.

related to changes in the contact state. Moreover, these transition tasks are parametrizable but provide a stereotypical reaction, the dynamics of which is pre-planned and that is thus not well suited to deal with very dynamic situations.

Similarly to the work in [7], one can take advantage of task and sensor information that is available to the robot. For example, when a humanoid walks towards a target, the desired walking pattern can be computed in advance [8,9]. Moreover, sensor data can be used to estimate when the contact may be established. In these scenarios, task and sensor information may allow the robot to act in advance to avoid large sudden changes in control signals. However, instead of using pre-planned strategies to manage contact transitions in a reactive controller, these information can be accounted for in a predictive way thus endowing the controller with both robustness and reactivity.

The goal of paper is to minimise the rate of change in torques during the addition and removal of the contact constraints using Model Predictive Control (MPC). Using a finite receding horizon, the MPC can preview sudden changes in contacts in advance and have time to react to smooth them. In practice, the MPC produces a time-varying maximum and minimum allowable contact force constraint that is added into the QP control scheme. This MPC generated constraint is computed such that its rate of change is minimised. This extra constraint in the QP control scheme reduces abrupt changes of the actual contact forces, resulting in less sudden changes in actuation torques. To demonstrate the effectiveness of the proposed approach, simulations for different scenarios are shown on the iCub humanoid robot. The results show that the proposed approach is able to reduce the sudden changes in torque significantly.

The remainder of the paper is organized as follows. Section II presents the reactive whole-body motion control framework. Section III proposes a smooth contact force generation approach using MPC. Section IV presents and discusses the simulation 
results. Finally, Section V concludes the paper and presents future research directions.

\section{REACTIVE CONTROL FRAMEWORK}

The equation of motion of a free-floating system with $n$ degrees of freedom (DoF) of joints and 6 DoF of free-floating base can be derived from the Euler-Lagrange formalism and expressed as:

$$
M(\boldsymbol{q}) \underbrace{\left[\begin{array}{c}
\dot{\boldsymbol{\nu}}_{b} \\
\ddot{\boldsymbol{q}}_{j}
\end{array}\right]}_{\dot{\boldsymbol{\nu}}}+\boldsymbol{n}(\boldsymbol{q}, \boldsymbol{\nu})=S \boldsymbol{\tau}+J_{c}(\boldsymbol{q})^{T} \boldsymbol{F}_{c},
$$

where $\boldsymbol{q} \in \mathbb{R}^{n+6}$ parametrizes the configuration of the freefloating system. $\boldsymbol{q}_{j} \in \mathbb{R}^{n}$ parametrizes the joint configuration in the joint space. $\nu \in \mathbb{R}^{n+6}$ represents the system velocities, concatenating the floating-base twist $\nu_{b}$ and the joint velocities $\dot{\boldsymbol{q}}_{j} \cdot \dot{\boldsymbol{\nu}}=\left[\begin{array}{ll}\dot{\boldsymbol{\nu}}_{b} & \ddot{\boldsymbol{q}}_{j}\end{array}\right]^{T}$ is the system accelerations. The matrix $M(\boldsymbol{q}) \in \mathbb{R}^{(n+6) \times(n+6)}$ is the generalized inertia matrix, and $\boldsymbol{n}(\boldsymbol{q}, \boldsymbol{\nu}) \in \mathbb{R}^{n+6}$ is the vector of Coriolis, centrifugal and gravity terms. The term $S$ is the actuation matrix for the joint torque vector $\tau \in \mathbb{R}^{n}$ [10]. The matrix $J_{c}(\boldsymbol{q})$ is the Jacobian matrix at the contact point, and $\boldsymbol{F}_{c}$ is the external contact force. The tangential and normal components of $\boldsymbol{F}_{c}$ are denoted by $\boldsymbol{F}_{t}$ and $\boldsymbol{F}_{n}$, respectively, with $\boldsymbol{F}_{n}=F_{n} \boldsymbol{n}$ and $\boldsymbol{n}$ being the normal vector to the contact surface. The action variable is defined as $\boldsymbol{\chi}=\left[\begin{array}{lll}\dot{\boldsymbol{\nu}}^{T} & \boldsymbol{\tau}^{T} & \boldsymbol{F}_{c}^{T}\end{array}\right]^{T}$.

\section{A. Contact Constraints}

Contact constraints require two conditions to be satisfied in order to maintain contact between two objects. One is the contact existing constraint, and the other is the friction cone constraint.

1) Contact Existing Constraint: Assuming that the environment is static and rigid, when a contact is established, the contact existing constraint can be expressed as follows:

$$
\begin{gathered}
\ddot{\boldsymbol{x}}_{c}=J_{c}(\boldsymbol{q}) \dot{\boldsymbol{\nu}}+\dot{J}_{c}(\boldsymbol{q}, \boldsymbol{\nu}) \boldsymbol{\nu}=0, \\
F_{n} \geq 0 .
\end{gathered}
$$

2) Friction Cone Constraint: For a non-sliding contact, the contact force for each contact point is constrained within the Coulomb friction cone:

$$
\left\|\boldsymbol{F}_{t}\right\| \leq \mu\left\|\boldsymbol{F}_{n}\right\|
$$

where $\mu$ is the friction coefficient. The friction cone can be approximated by a polygonal cone expressed as a set of linear inequality constraints [11]:

$$
C \boldsymbol{F}_{c} \leq 0
$$

\section{B. Allowable Force Limits}

Contact forces can be constrained by various contact constraints according to different scenarios. For example, when there is no contact, the contact force must be 0 ; when a robot stands on one foot, the contact force must be at least the force of gravity; and this contact force is required to be limited in order to avoid damage to the environment, or to be larger than a certain value to be able to manipulate an object. In this case, constraint (3) can be modified by adding a maximum allowable force $F_{\max } \geq 0$ and a minimum allowable force $F_{\min } \geq 0$ :

$$
F_{\min } \leq F_{n} \leq F_{\max },
$$

where $F_{\max }$ and $F_{\min }$ are both related to the scenario.

\section{Quadratic Program Control Scheme}

A Quadratic Program (QP) [7] can be applied to resolve the action variable $\chi$ by optimizing a cost function associated with a set of tasks $T(\chi)$ while satisfying the whole-body dynamics equation (1) as well as the contact constraints (2), (5) and (6):

$$
\begin{array}{cc}
\boldsymbol{\chi}^{*}=\underset{\boldsymbol{\chi}}{\arg \min } & \|T(\boldsymbol{\chi})\|_{Q}^{2}+\|\boldsymbol{\chi}\|_{R}^{2} \\
\text { s.t } & M(\boldsymbol{q}) \dot{\boldsymbol{\nu}}+\boldsymbol{n}(\boldsymbol{q}, \boldsymbol{\nu})=S \boldsymbol{\tau}+J_{c}(\boldsymbol{q})^{T} \boldsymbol{F}_{c} \\
J_{c}(\boldsymbol{q}) \dot{\boldsymbol{\nu}}+\dot{J}_{c}(\boldsymbol{q}, \boldsymbol{\nu}) \boldsymbol{\nu}=0 \\
C \boldsymbol{F}_{c} \leq 0 \\
F_{\text {min }} \leq F_{n} \leq F_{\max }
\end{array}
$$

where $Q$ and $R$ are diagonal weighting matrices that govern the priority between the task and the control effect [7]. The reactive QP control scheme (7) has to instantaneously adapt action variables to variations of $F_{\max }$ and $F_{\min }$. But such variations of force limits could be abrupt. For example, when a contact is established or broken suddenly, large sudden changes in actuation torques can be generated. Therefore, this control scheme is improved in Section III to minimise the rate of changes in actuation torques.

\section{MPC CONTACT Force Constraint Generation}

\section{A. General Concept}

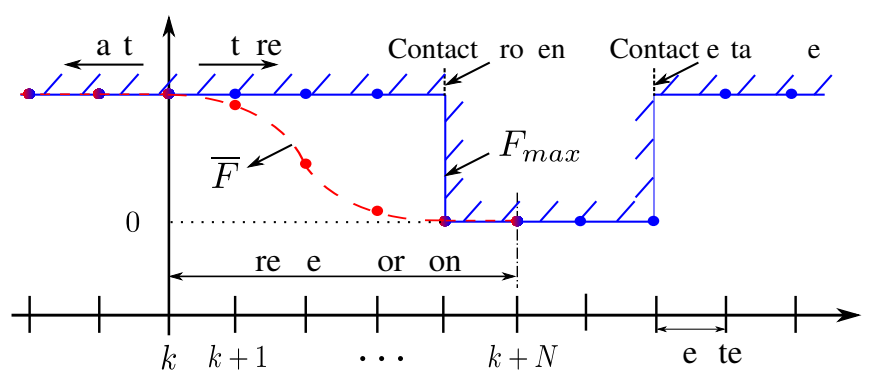

Fig. 2: Smoothing the evolution of the maximum allowable force using the MPC at time step $k$.

In this paper, Model Predictive Control (MPC) [12,13] is used to reduce the large sudden changes of $F_{\max }$ and $F_{\min }$ online with the goal to produce less sudden changes in actuations torques. The proposed MPC scheme is shown in Figure 2. Given the evolution of $F_{\max }$ for example, its changes can be known in advance and accounted for by the MPC through a preview window of $N$ steps with a sampling time $T$. The aim of the MPC is to generate smooth maximum allowable force $\bar{F}$ and smooth minimum allowable force $\underline{F}$ with less changes. Then, it can replace $F_{\max }$ and $F_{\min }$ in constraint (6):

$$
\underline{F} \leq F_{n} \leq \bar{F} .
$$

The following part of this section explains the computation of $\bar{F}$ and $\underline{F}$.

\section{B. MPC Smooth Allowable Force Generation}

A discrete-time linear model of the force can be expressed as:

$$
\begin{aligned}
& \bar{F}_{k+1}=\bar{F}_{k}+\dot{\bar{F}}_{k} T, \\
& \underline{F}_{k+1}=\underline{F}_{k}+\underline{\dot{F}}_{k} T,
\end{aligned}
$$

where $\bar{F}_{k}$ and $\underline{F}_{k}$ are the maximum allowable force and the minimum allowable force at time step $k$, respectively. $\dot{\bar{F}}_{k}$ and 


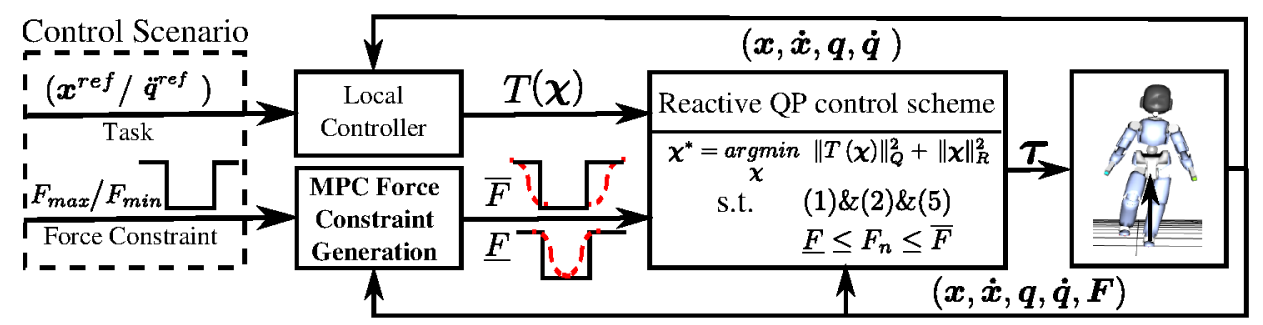

Fig. 3: Block diagram of the control framework.

$\underline{\underline{F}}_{k}$ are the first-order time derivative of $\bar{F}_{k}$ and $\underline{F}_{k}$, respectively. $\bar{F}_{k}$ and $\underline{F}_{k}$ should be optimized according to the original profile of $F_{\max }$ and $F_{\min }$. Within the time horizon $N T$, the MPC for generating a optimized $\bar{F}$ and $\underline{F}$ can be formulated as:

$$
\begin{aligned}
& \begin{array}{ll}
\underset{\dot{\bar{F}}_{k, \ldots, k+N}, \underline{\underline{F}}_{k, \ldots, k+N}}{\arg \min } & \sum_{j=k}^{k+N}\left\|\bar{F}_{j}-F_{\max , j}\right\|^{2}+\alpha\left\|\dot{\bar{F}}_{j}\right\|^{2} \\
& +\left\|\underline{F}_{j}-F_{\min , j}\right\|^{2}+\alpha\left\|\dot{\bar{F}}_{j}\right\|^{2}
\end{array} \\
& \text { subject to } \quad \bar{F}_{k+1}=\bar{F}_{k}+\dot{\bar{F}}_{k} T \\
& \underline{F}_{k+1}=\underline{F}_{k}+\underline{\dot{F}}_{k} T \\
& F_{\min , j} \leq \underline{F}_{j} \leq \bar{F}_{j} \leq F_{\max , j}
\end{aligned}
$$

where (11d) ensures that at each time step $j$ every solution $\chi^{*}$ that satisfies the constraint (8) also satisfies the constraint (6). The coefficient $\alpha$ is the weight, governing the importance between the changes of $\bar{F}$ and the deviation from the original maximum allowable force. $\alpha$ also has the same effect on $\underline{F}$. For example, a higher value of $\alpha$ enhances the reduction of the changes of $\bar{F}$; however, the constraint (8) may turn to be more conservative and actuation capacities may be restricted. In (11), at time step $k, \dot{\bar{F}}_{k}^{*}$ and $\underline{F}_{k}^{*}$ can be computed by a Linear Quadratic Program solver [14]. The generated maximum allowable force is $\bar{F}_{k}=$ $\bar{F}_{k-1}+\bar{F}_{k}^{*} T$ and the generated minimum allowable force is $\underline{F}_{k}=\underline{F}_{k-1}+\underline{\dot{F}}_{k}^{*} T$.

The overall control framework is shown in Figure 3. The proposed MPC, as an online approach, generates smooth evolution of the maximum allowable force $\bar{F}$ and the minimum allowable force $\underline{F}$ with less sudden changes. The results of the MPC is used by the reactive QP control scheme to minimise large sudden changes in actuation torques. To achieve this, the baseline reactive QP control scheme (7) is improved by implementing the maximum and minimum allowable force provided by the MPC as follows:

$$
\begin{array}{cc}
\boldsymbol{\chi}^{*}=\underset{\boldsymbol{\chi}}{\arg \min } & \|T(\boldsymbol{\chi})\|_{Q}^{2}+\|\boldsymbol{\chi}\|_{R}^{2} \\
\text { s.t } & M(\boldsymbol{q}) \dot{\boldsymbol{\nu}}+\boldsymbol{n}(\boldsymbol{q}, \boldsymbol{\nu})=S \boldsymbol{\tau}+J_{c}(\boldsymbol{q})^{T} \boldsymbol{F}_{c} \\
J_{c}(\boldsymbol{q}) \dot{\boldsymbol{\nu}}+\dot{J}_{c}(\boldsymbol{q}, \boldsymbol{\nu}) \boldsymbol{\nu}=0 \\
C \boldsymbol{F}_{c} \leq 0 \\
\underline{F} \leq F_{n} \leq \bar{F}
\end{array}
$$

To illustrate the proposed MPC approach, a simple example of generating a smooth evolution of $\bar{F}$ given a discontinuous $F_{\max }$ is provided with $T=0.01 \mathrm{~s}, \alpha=1.0 e-2$ and $N T=1.5 \mathrm{~s}$. In this example, $F_{\min }$ is 0 all the time. In Figure 4 , it can be observed that the generated maximum allowable force $\bar{F}$ evolves smoothly with less changes compared with the original $F_{\max }$. $\bar{F}$ can gradually decrease to zero before a contact is broken and

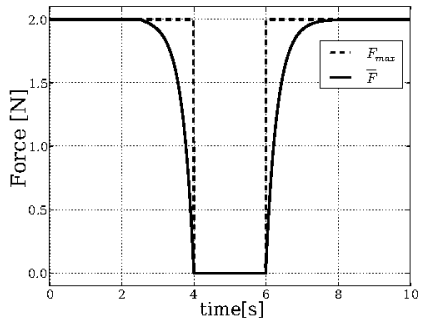

(a) Evolution of $\bar{F}$.

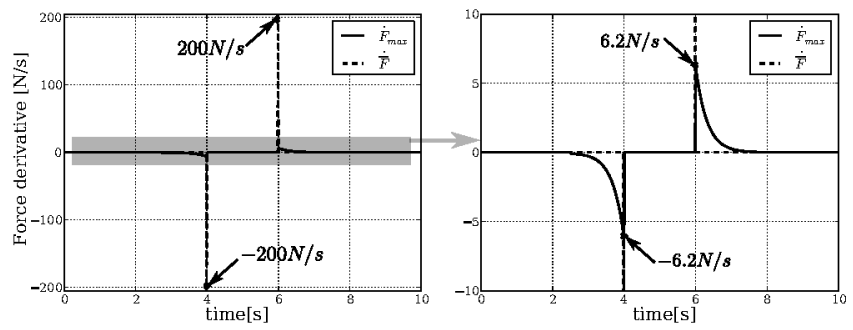

(b) Evolution of $\dot{\bar{F}}$.

Fig. 4: The resulting smooth constraint.

increase gradually after a contact is established. The MPC begins to reduce $\bar{F}$ at $t=2.5 \mathrm{~s}$ when it detects the changes of $F_{\max }$ in the preview window. At $t=4.0 \mathrm{~s}$ and $t=6.0 \mathrm{~s}$ when the large changes occur on $F_{\max }$, the magnitude of force derivatives is significantly reduced from $200 \mathrm{~N} / \mathrm{s}$ with the original evolution of force $F_{\max }$ to 6.2N/s with the MPC generated force $\bar{F}$.

Note that an alternative method to address this problem is to smooth the profile of $F_{\max }$ by applying a smooth polynomial spline $[15,16]$. However, the advantage of using MPC here is to be able to handle complex constraint profiles in an automatic and generic way to ensure that the optimized $\bar{F}$ constraint is compatible with $\underline{F}$ constraint as well (see Figure 5), whereas the use of smooth polynomial approaches require manual choose of the start and end points of the segment to be smoothed and manual tuning of polynomial parameters. Moreover, the use of polynomial to smooth each constraint may generate conflicts between one smoothed constraint and the other constraints, and may lead to oscillation problems [17] as well.

\section{SIMULATION RESULTS}

The proposed approach is applied to the control of a 38-DOF iCub robot using Arboris-Python simulator [18], an open-source dynamic simulator written by Python. The iCub robot is actuated by joint torques to perform tasks in operational space under contact constraints. The results of the proposed approach (12) are compared with those of the baseline approach (7). 


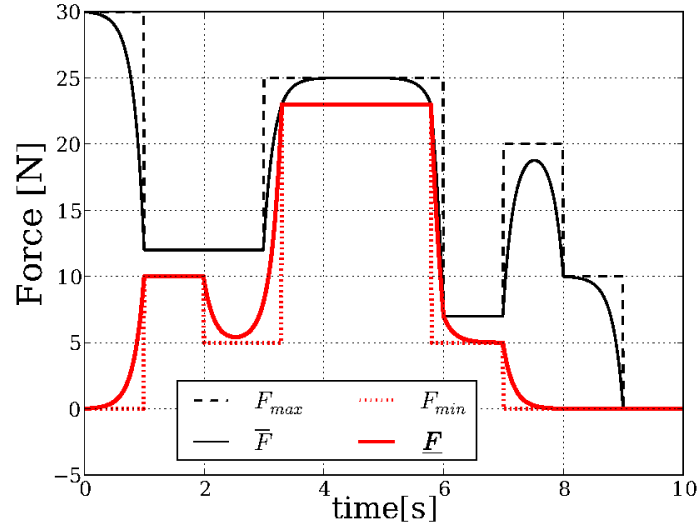

Fig. 5: Smoothing the evolution of the maximum and minimum allowable force simultaneously using the MPC.

\section{A. Standing up from a chair}

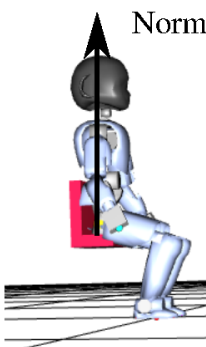

(1)

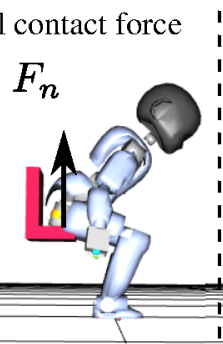

(2)

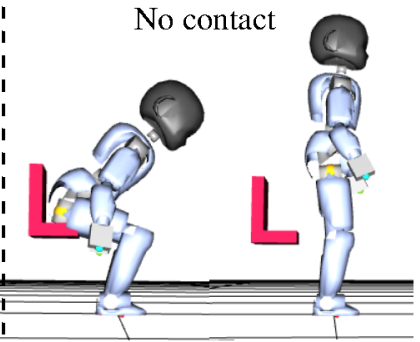

(3)

(4)

Fig. 6: Snapshots of four steps as the robot stands up from a chair.

Figure 6 illustrates the 4 steps required for the robot to stand up from a chair:

1) The robot is sitting on the chair and the contact with the chair exists;

2) The robot moves its CoM into the support polygon of the feet in preparation for leaving the chair;

3) The contacts between the robot and the chair is broken;

4) The robot stands up.

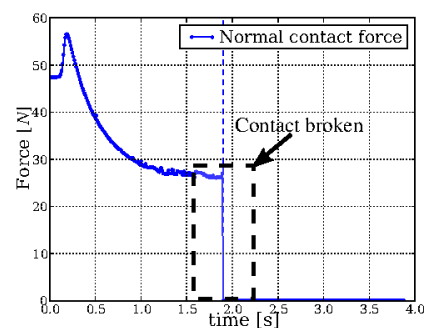

(a) The normal contact force decreases to zero abruptly when the contact is broken.

Fig. 7: Evolution of the normal contact force between the robot and the chair with the baseline approach (a) and the proposed approach (b).

The resulting evolutions of the normal contact force between the robot and the chair using the baseline approach and the proposed approach are shown in Figure 7(a) and Figure 7(b), respectively. The evolutions of the knee joint torque and its derivative are shown in Figure 8 for both approaches.

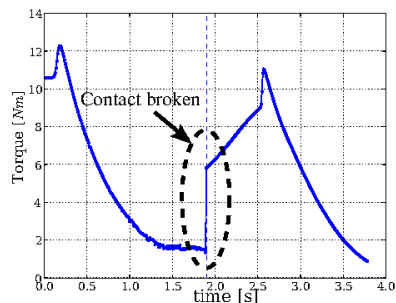

(a) A torque peak appears when the contact is broken.

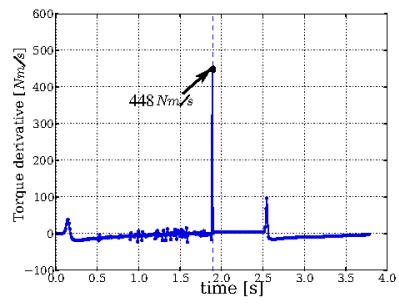

(c) The torque derivative reaches a maximum of $448 \mathrm{Nm} / \mathrm{s}$ when the contact is broken.

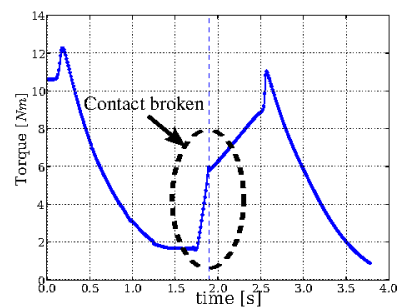

(b) The joint torque increases as expected in a smooth way.

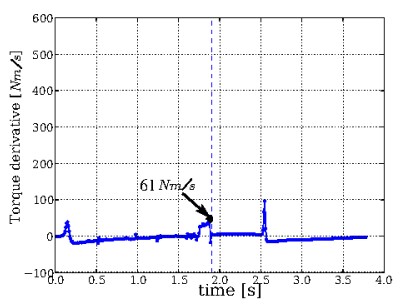

(d) The torque derivative is only about $61 \mathrm{Nm} / \mathrm{s}$.
Fig. 8: Evolution of the knee joint torque and its torque derivative with the baseline approach (left) and the proposed approach (right).

Using the baseline approach, when the contact constraint between the chair and the robot is removed at $t=1.9 \mathrm{~s}$, the normal contact force instantaneously becomes zero (see Figure 7(a)). Among all of the joints in the iCub, the knee joint exhibits the largest sudden changes in torques and its torque derivative is $448 \mathrm{Nm} / \mathrm{s}$ (see Figure 8(a) and (c)).

Using the proposed approach, the evolution of the normal contact force is shown in 7(b) with the time horizon $N T=0.5 \mathrm{~s}$ and a normal contact force of $F_{\max }=60 \mathrm{~N}$. Once the removal of the contact constraint is detected by the MPC at $0.5 \mathrm{~s}$ before it occurs, the MPC starts to decrease the contact force towards zero. The change of the contact force is significantly reduced at $t=1.9 \mathrm{~s}$ compared with the result using the baseline approach, when the contact is broken. Furthermore, the torque of the knee joint evolves with less changes and its torque derivative is only $61 \mathrm{Nm} / \mathrm{s}$ (see Figure 8(d)).

\section{B. Lifting and putting down the foot}

In this scenario, the robot lifts its right foot off the ground at $t=2.0 \mathrm{~s}$ and then puts it back down at $t=5.0 \mathrm{~s}$. The action includes five steps as shown in Figure 9:

1) The robot is standing on the ground;

2) The robot moves its CoM above the left foot to ensure that it doesn't fall down after the contact of the right foot is broken;

3) The contact of the right foot is broken and the robot lifts it;

4) The robot puts down the right foot and the contact is reestablished;

5) The robot goes back to initial posture.

Figure 10 shows the evolutions of the normal contact forces on both feet using the baseline approach and the proposed approach. 


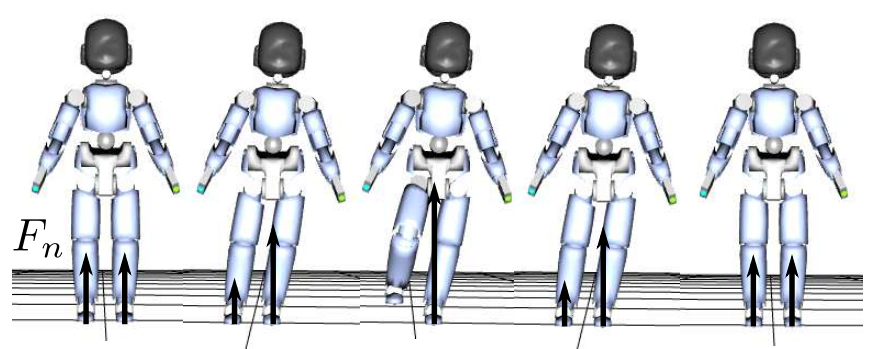

Fig. 9: Snapshots of lifting and putting down the right foot with 5 steps.

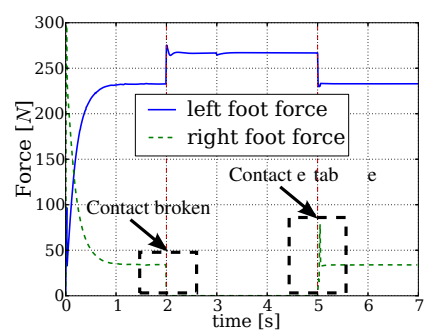

(a) Large changes occur on the contact force of feet when the contact is broken and established.

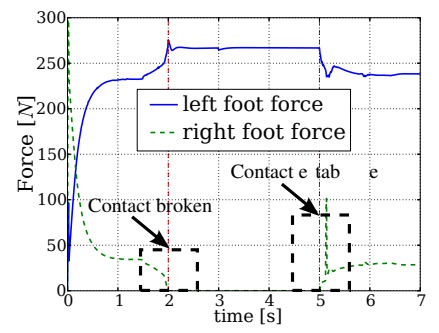

(b) No large changes occur on the contact force of feet when the contact is broken and established.

Fig. 10: Evolution of normal contact force on feet with the baseline approach (a) and the proposed approach (b).

Figure 11 shows the resulting evolutions of the knee joint torque and its derivative for both approaches.

Using the baseline approach, Figure 10(a) shows that the normal contact force changes abruptly at $t=2.0 \mathrm{~s}$ and $t=5.0 \mathrm{~s}$ when the right foot contact is broken and established, respectively. Once the right foot lifts off the ground, the whole-body weight shifts from the double feet support to one foot support. The sudden decrease of the normal contact force on the right foot directly leads to the increase of the normal contact force on the left foot. These sudden changes of contact forces result in the discontinuities in actuation torques. The hip joint exhibits the largest changes in torques among all of the joints and its torque derivative is up to $920 \mathrm{Nm} / \mathrm{s}$ (shown in Figure 11(a) and (c)).

Using the proposed approach with time horizon $N T=0.5 \mathrm{~s}$, the normal contact force of the right foot gradually decreases to zero before the contact is broken and smoothly increases after the contact is added (see Figure 10(b)). As a result, large changes in the contact force are avoided, and as shown in Figures 11(b) and (d), no large changes in hip torques occur. The torque derivative is significantly reduced from $920 \mathrm{Nm} / \mathrm{s}$ using the baseline approach to $85 \mathrm{Nm} / \mathrm{s}$ using the proposed approach.

In Figure 10, it is observed that a spike appears around $t=$ $5.0 \mathrm{~s}$. This spike is the impact force due to the collision between two rigid objects. Reducing this impact force peak would require to locally adapt the apparent impedance of the foot making contact $[19,20]$. This problem is not addressed by the proposed approach.

\section{Limitations}

In order to verify the effectiveness of the proposed approach in a more dynamic situation, the proposed approach is extended to the scenario of walking. In this scenario, the desired walking pattern can be computed by ZMP planning [9]. The robot spends

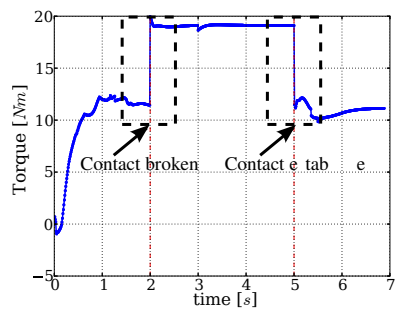

(a) Torque peaks appear when the contact is broken and established.

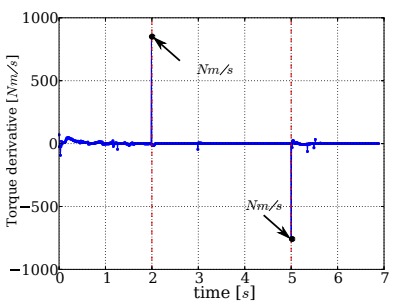

(c) The torque derivatives reaches a absolute value maximum of $920 \mathrm{Nm} / \mathrm{s}$ when the contact is broken and established.

Fig. 11: Evolution of hip joint torque and its torque derivative with the baseline approach (left) and the proposed approach (right).

one second to move one step and it has $0.3 \mathrm{~s}$ for double support. The maximum allowable force is $300 \mathrm{~N}$.

Figures 12(a) and (b) show the evolutions of the normal contact force on both feet using the baseline approach and the proposed approach, respectively. Figures 12(c) and (d) show the hip joint torque and its derivative for both approaches, respectively.

Figure 12 shows that the changes of the normal contact forces and joint torques by using the proposed approach (12(b) and (d)) are only slightly reduced compared to those using the baseline approach (12(a) and (c)). This is because in dynamic walking, the whole-body weight shifts quickly between double feet support and one foot support. A large maximum allowable contact force $F_{\max }$ is required to support the heavy robot with one foot. Moreover, dynamic walking requires fast contact modifications. Each foot has a short time in contact with the ground between lifting and lowering phases. Indeed, the proposed scheme must decrease a large maximum force to zero in a short time, resulting in a large change of the contact force at the moment when the contact is broken. Therefore, the effects of the proposed approach are limited in very dynamic situations requiring a large variation of the force in a short time.

Nevertheless, the proposed approach has three main advantages. First, prediction allows smooth actuation torques to be generated in situations where purely reactive approaches would fail to do so. Although global planning provides such a smoothing feature, prediction over a finite receding horizon can be seen as "short term reactive" and is thus better suited for dynamically changing situations. Second, the concept can be generalized to any constraint, the evolution of which can be known a priori (e.g. obstacle avoidance). Finally, the proposed method acts only on the constraints and can be directly used within any control scheme that handles constraints as inequalities. 


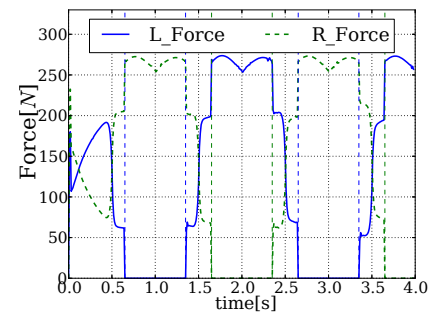

(a) Evolution of feet forces using the baseline approach.
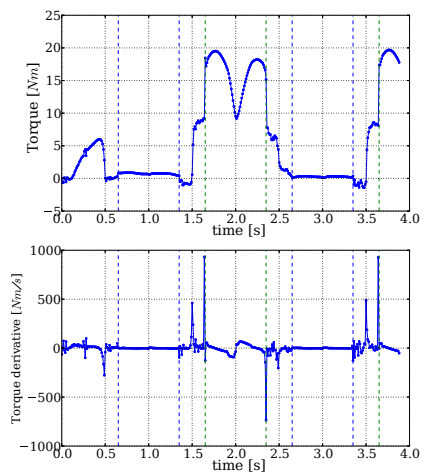

(c) Evolution of the hip joint torque (top) and its derivative (bottom) using the baseline approach.

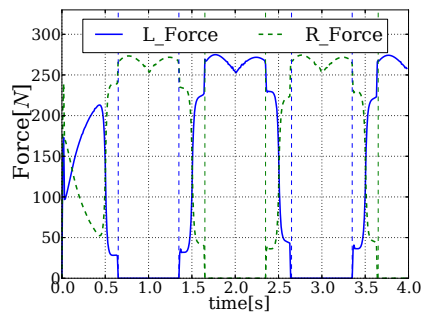

(b) Evolution of feet forces using the proposed approach.
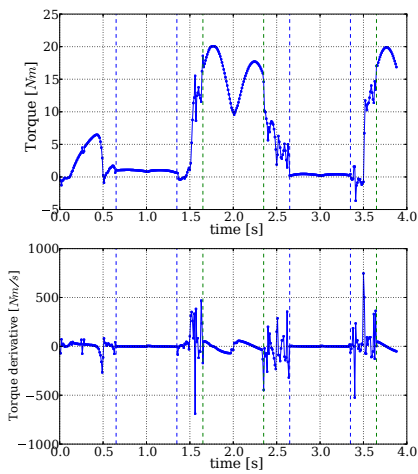

(d) Evolution of the hip joint torque (top) and its derivative (bottom) using the proposed approach.

Fig. 12: Evolution of hip joint torque and its torque derivative with the baseline approach (left) and the proposed approach (right).

\section{CONCLUSION}

In this paper, the proposed MPC approach is used to minimise the rate of change in actuation torques. The contribution here is to endow a reactive control scheme, which handles constraints as inequalities, with the ability to anticipate and adapt more robustly to changes of contacts.

The proposed MPC can preview sudden changes in contacts over a finite receding horizon and generate a smooth contact force constraint, the changes of which are minimised. The reactive QP control scheme uses this MPC generated constraint to reduce the sudden changes of the actual contact forces. As a result, the rate of change in actuation torques is minimised. Simulations involving breaking and establishing contacts show that the proposed approach can successfully minimise instantaneous changes in actuation torques. The results in walking situations also show that the proposed approach has limitations related to the dynamics of the motions to be performed.

Future work will focus on analysing the influence of the length of the preview window and the weight $\alpha$ in the MPC scheme on the performances of the proposed scheme. Implementation on the iCub robot is also envisioned.

\section{ACKNOWLEDGMENT}

This work was partially supported by the European Commission, within the CoDyCo project (FP7-ICT-2011-9, No.600716), by BPIFrance through the Romeo2 project and by the RTE company through the RTE/UPMC chair Robotics Systems for field intervention in constrained environments held by Vincent Padois.

\section{REFERENCES}

[1] Y. Abe, M. da Silva, and J. Popović, "Multiobjective control with frictional contacts," in Proceedings of the ACM SIGGRAPH/Eurographics symposium on Computer animation, 2007, pp. 249-258.

[2] P. M. Wensing and D. Orin, "Generation of dynamic humanoid behaviors through task-space control with conic optimization," in Proceedings of the IEEE International Conference on Robotics and Automation, 2013, pp. 3103-3109.

[3] A. Herzog, L. Righetti, F. Grimminger, P. Pastor, and S. Schaal, "Balancing experiments on a torque-controlled humanoid with hierarchical inverse dynamics," in Proceedings of the IEEE/RSJ International Conference on Intelligent Robots and Systems, 2014, pp. 981-988.

[4] C. Klein and S. Kittivatcharapong, "Optimal force distribution for the legs of a walking machine with friction cone constraints," IEEE Transactions on Robotics and Automation, vol. 6, no. 1, pp. 73-85, 1990.

[5] U. Muico, Y. Lee, J. Popović, and Z. Popović, "Contact-aware nonlinear control of dynamic characters," ACM Transactions on Graphics, vol. 28 , no. 3 , p. 81,2009 .

[6] J.-S. Pang and J. C. Trinkle, "Complementarity formulations and existence of solutions of dynamic multi-rigid-body contact problems with coulomb friction," Mathematical programming, vol. 73, no. 2, pp. 199-226, 1996.

[7] J. Salini, V. Padois, and P. Bidaud, "Synthesis of complex humanoid whole-body behavior: a focus on sequencing and tasks transitions," in Proceedings of the IEEE International Conference on Robotics and Automation, 2011, pp. 1283-1290.

[8] M. Vukobratović and B. Borovac, "Zero-moment pointthirty five years of its life," International Journal of Humanoid Robotics, vol. 1, no. 01, pp. 157-173, 2004.

[9] S. Kajita, F. Kanehiro, K. Kaneko, K. Fujiwara, K. Harada, K. Yokoi, and $\mathrm{H}$. Hirukawa, "Biped walking pattern generation by using preview control of zero-moment point," in Proceedings of the IEEE International Conference on Robotics and Automation, vol. 2, 2003, pp. 16201626.

[10] J. Salini, "Dynamic control for the task/posture coordination of humanoids: toward synthesis of complex activities," Thèse de doctorat Université Pierre et Marie Curie, Paris, France, 2012.

[11] I. Kao, K. Lynch, and J. Burdick, Springer Handbook of Robotics. Springer, 2008, ch. 27. Contact Modeling and Manipulation.

[12] J. Bellingham, Y. Kuwata, and J. How, "Stable receding horizon trajectory control for complex environments," in Proceedings of the AIAA Guidance, Navigation, and Control Conference, 2003.

[13] E. F. Camacho and C. B. Alba, Model predictive control. Springer, 2013

[14] J. Nocedal and S. J. Wright, Numerical Optimization, Second Edition. Springer New York, 2006

[15] C.-S. Lin, P.-R. Chang, and J. Y. S. Luh, "Formulation and optimization of cubic polynomial joint trajectories for industrial robots," IEEE Transactions on Automatic Control, vol. 28, no. 12, pp. 1066-1074, 1983.

[16] P. Lambrechts, M. Boerlage, and M. Steinbuch, "Trajectory planning and feedforward design for high performance motion systems," in Proceedings of the American Control Conference, vol. 5, 2004, pp. 4637-642.

[17] M. Schatzman, Numerical Analysis: a Mathematical Introduction. Oxford University Press, 2002.

[18] J. Salini, S. Barthélemy, and A. Micaelli, "Arboris-python." [Online]. Available: http://www.isir.upmc.fr/?op=view_profil\&lang= fr\&id=121\&pageid $=766$

[19] I. D. Walker, "The use of kinematic redundancy in reducing impact and contact effect in manipulation," in Proceedings of the IEEE InternationalConference on Robotics and Automation, 1990, pp. 434 439.

[20] P. Pagilla and B. Y. B. Yu, "A stable transition controller for constrained robots," IEEE/ASME Transactions on Mechatronics, vol. 6 , no. 1 , pp. $65-74,2001$ 